\title{
Эпидемиологические аспекты желудочковых нарушений ритма сердца у молодых пациентов после инфаркта миокарда в условиях амбулаторной реабилитации
}

\author{
И.А. Новикова ${ }^{1}$, Л.А. Некрутенко ${ }^{2}$, Л.М. Василец ${ }^{2}$, Е.А. Шишкина ${ }^{2}$, Р.А. Родионов ${ }^{2}$ \\ ${ }^{1}$ Федеральный центр сердечно-сосудистой хирургии имени С.Г. Суханова, \\ Россия, 614013, г. Пермь, ул. Маршала Жукова, д. 35 \\ ${ }^{2}$ Пермский государственный медицинский университет имени академика Е.А. Вагнера, \\ Россия, 614000, г. Пермь, ул. Петропавловская, 26 \\ E-mail: nurdus@yandex.ru
}

\begin{abstract}
Аннотация. Инфаркт миокарда является основной причиной систолической сердечной недостаточности. Желудочковые нарушения ритма - одна из наиболее частых причин внезапной сердечной смерти у таких пациентов. Цель исследования - изучить эпидемиологические особенности желудочковых аритмий у молодых пациентов, перенесших инфаркт миокарда, на амбулаторном этапе реабилитации. Обследовано 108 пациентов в возрасте от 18 до 45 лет, перенесших инфаркт миокарда в период с 01.01.2017 по 01.01.2019. Всем пациентам через 12 месяцев после инфаркта миокарда были проведены эхокардиографическое исследование и мониторирование ЭКГ по Холтеру. По данным эхокардиографического исследования через 12 месяцев после инфаркта миокарда $60 \%$ молодых пациентов имеют диастолическую, а $18 \%$ - систолическую дисфункцию, у $42 \%$ сохраняются зоны локальной асинергии, у $16 \%$ формируется хроническая аневризма сердца. У каждого пятого молодого пациента наблюдается дезадаптивный тип ремоделирования сердца - эксцентрическая гипертрофия левого желудочка. У молодых пациентов на амбулаторном этапе реабилитации отмечается высокая среднесуточная частота сердечных сокращений, частая желудочковая экстрасистолия регистрируется у 30 \% пациентов, а эпизоды неустойчивой желудочковой тахикардии - у каждого десятого. В 12,5 \% случаев сохраняются эпизоды ишемической депрессии ST. Результаты исследования представляют негативный прогноз у молодых больных после перенесенного инфаркта миокарда.
\end{abstract}

Ключевые слова: инфаркт миокарда, молодой возраст, желудочковая экстрасистолия, желудочковая тахикардия, амбулаторная реабилитация, ремоделирование сердца.

Для цитирования: Новикова И.А., Некрутенко Л.А., Василец Л.М., Шишкина Е.А., Родионов Р.А. 2020. Эпидемиологические аспекты желудочковых нарушений ритма сердца у молодых пациентов после инфаркта миокарда в условиях амбулаторной реабилитации. Актуальные проблемы медицины, 43 (3): 383-391. DOI: 10.18413/2687-0940-2020-43-3-383-391

\section{Epidemiological features of ventricular rhythm disorders In young post myocardial infarction patients under conditions of ambulatory rehabilitation}

\author{
Irina A. Novikova ${ }^{1}$, Ludmila A. Nekrutenko ${ }^{2}$, Liubov M. Vasilets ${ }^{2}$, \\ Ekaterina A. Shishkina ${ }^{2}$, Roman A. Rodionov ${ }^{2}$ \\ ${ }^{1}$ S. Sukhanov Federal Center for Cardiovascular Surgery, \\ 35 Marshal Zhukov st., Perm, 614013, Russia \\ ${ }^{2}$ Academician Ye.A. Wagner Perm State Medical University, \\ 26 Petropavlovskaya st., Perm, 614000, Russia \\ E-mail: nurdus@yandex.ru
}

Abstract. Myocardial infarction is the main cause of systolic heart failure. Ventricular arrhythmia is one of the most common causes of sudden cardiac death in such patients. The purpose of the 
study was to examine the epidemiological features of ventricular arrhythmias in young post myocardial infarction patients at the ambulatory stage of rehabilitation. We examined 108 patients aged 18 to 45 years who experiend myocardial infarction from 01.01.2017 to 01.01.2019. One year after myocardial infarction all patients underwent echocardiography and cardiac rhythm monitoring. According to the echocardiography data $60 \%$ of young patients have diastolic dysfunction, $18 \%$ - systolic dysfunction, $42 \%$ - left ventricular asynergy, $16 \%$ - heart wall aneurysm. In $20 \%$ of cases a maladaptive type of heart remodeling is observed. In young patients at the ambulatory stage of rehabilitation a high average daily heart rate is noted. Frequent premature ventricular contractions are recorded in $30 \%$ of patients and 9,1\% had episodes of ventricular tachycardia. The study results represent negative prognosis in young post myocardial infarction patients.

Keywords: myocardial infarction, young age, premature ventricular contractions, ventricular tachycardia, ambulatory rehabilitation, heart remodeling.

For citation: Novikova I.A., Nekrutenko L.A., Vasilets L.M., Shishkina E.A., Rodionov R.A. 2020. Epidemiological features of ventricular rhythm disorders in young post myocardial infarction patients under conditions of ambulatory rehabilitation. Challenges in Modern Medicine, 43 (3): 383-391 (in Russian). DOI: 10.18413/2687-0940-2020-43-3-383-391

\section{Введение}

Несмотря на то, что в последние годы прогноз у пациентов, перенесших инфаркт миокарда (ИМ), значительно улучшился, ишемическая болезнь сердца (ИБС) продолжает занимать ведущую позицию в структуре причин смертности в мире [Setoguchi et al., 2008; World Health Organization, 2014]. Как в развитых, так и в развивающихся странах ИМ является основной причиной систолической сердечной недостаточности [Chang et al., 2019]. Желудочковые нарушения ритма - одна из наиболее частых причин внезапной сердечной смерти (BCC) у пациентов с ИМ и сердечной недостаточностью [Tomaselli, Zipes, 2004; McMurray et al., 2005]. Установлено, что в постинфарктном периоде наличие желудочковых аритмий увеличивает риск повторных неблагоприятных сердечно-сосудистых событий [Jons et al., 2019]. Частота ИМ в молодом возрасте относительно невелика, однако в последние годы она стремительно растет. По сравнению со здоровыми молодыми людьми риск ВСС у молодых пациентов, перенесших инфаркт миокарда, повышается в 74 раза [Risgaard et al., 2013]. Большая часть летальных исходов происходит в течение первого года после ИМ [Solomon et al., 2005; Пракопчик, Гуревич, 2008]. В связи с вышеизложенным изучение эпидемиологических особенностей желудочковых нарушений ритма среди пациентов молодого возраста в реабилитационном периоде ИМ представляется чрезвычайно актуальным.

Цель исследования - изучить эпидемиологические особенности желудочковых аритмий у молодых пациентов, перенесших ИМ, на амбулаторном этапе реабилитации.

\section{Объекты и методы исследования}

Было обследовано 108 пациентов в возрасте от 18 до 45 лет, перенесших ИМ в период с 01.01.2017 по 01.01.2019. Критериями исключения из исследования были острые и хронические заболевания в стадии обострения, анемия средней и тяжелой степени, тяжелые нарушения функции печени и почек, системные заболевания. Исходная характеристика участников исследования представлена в таблице 1. 
Характеристика участников исследования Characteristics of patients

\begin{tabular}{|c|c|}
\hline Показатель & Молодые пациенты с ИМ (n = 108) \\
\hline Средний возраст Ме (Q1-Q3) & $41,0(38,0-43,0)$ \\
\hline Пол, \% & $\begin{array}{l}\text { мужчины 85,2 } \\
\text { женщины } 14,8\end{array}$ \\
\hline ИМпSТ, \% & 84,3 \\
\hline ИМбпSТ, $\%$ & 15,7 \\
\hline Индекс массы тела, кг/м² Me (Q1-Q3) & $27,0(24,4-30,0)$ \\
\hline Курение, \% & 65,4 \\
\hline Артериальная гипертензия, \% & 58,8 \\
\hline Отягощенная по ранней ИБС наследственность, \% & 54,6 \\
\hline Сахарный диабет 2 типа, \% & 9,8 \\
\hline Дислипидемия, \% & 58,9 \\
\hline
\end{tabular}

Большую часть молодых пациентов с ИМ составили лица мужского пола. Преобладал ИМ с подъемом сегмента ST (ИМпST). Молодые пациенты имели большую распространенность традиционных факторов риска сердечно-сосудистых заболеваний - курения, дислипидемии, артериальной гипертензии, отягощенной по ранней ИБС наследственности. Наиболее часто у молодых пациентов встречалась переднебоковая локализация инфаркта миокарда (рис. 1).

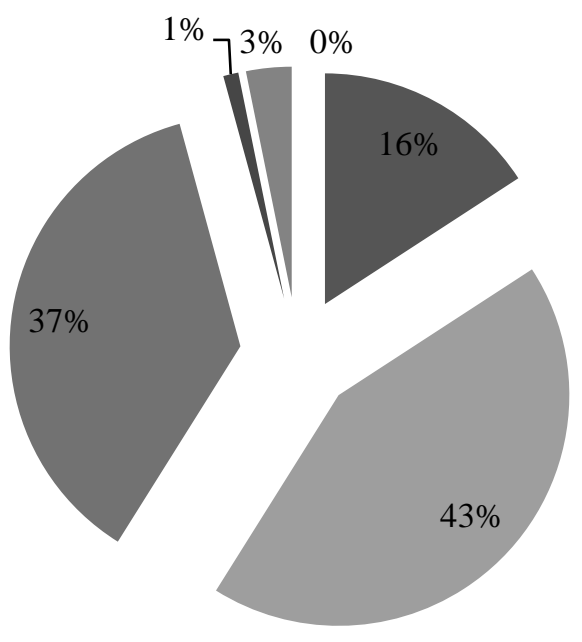

$$
\begin{aligned}
& \text { — Передний } \\
& \text { Передне-боковой } \\
& \text { — Задний } \\
& \text { - Задне-боковой } \\
& \text { — Циркулярный } \\
& \text { - Задний с вовлечением } \\
& \text { правого желудочка }
\end{aligned}
$$

Рис.1. Локализация инфаркта миокарда у молодых пациентов

Fig. 1. Localization of myocardial infarction in young patients

При поступлении в клинику всем пациентам была проведена коронароангиография. Стентирование коронарных артерий было выполнено в 71 \% случаев.

На амбулаторном этапе все пациенты получали двойную антитромбоцитарную терапию. Бета-адреноблокаторы были назначены 96,2 \% больных, статины - 95,2 \%, блокаторы ренин-ангиотензин-альдостероновой системы - 72,6 \%, антагонисты минералокортикоидных рецепторов - 23,1\%.

Всем пациентам, включенным в исследование, через 12 месяцев после ИМ были проведены эхокардиографическое исследование и мониторирование ЭКГ по Холтеру 
(ХМ-ЭКГ). В рамках ХМ-ЭКГ оценивалась суточная динамика частоты сердечных сокращений (ЧСС), нарушения ритма и проводимости, динамика сегмента ST.

Протокол исследования был одобрен Локальным этическим комитетом при Пермском государственном медицинском университете имени академика Е.А. Вагнера МЗ РФ в соответствии с Хельсинкской декларацией (2008). Все пациенты, включенные в исследование, подписали добровольное информированное согласие на участие в нем.

Статистическая обработка данных проводилась на базе пакета прикладной программы Microsoft Office Excel 2016 и пакета прикладных статистических программ IBM SPSS Statistics v.23 с использованием методов параметрического и непараметрического анализа. Количественные показатели в случае нормального распределения представлены как среднее арифметическое \pm стандартное отклонение, при отсутствии нормального распределения - при помощи медианы и нижнего и верхнего квартилей. Номинальные данные описывались с указанием процентных долей.

\section{Результаты и обсуждение}

По данным эхокардиографического исследования через 12 месяцев после ИМ $60 \%$ молодых пациентов имеют диастолическую, а $18 \%$ - систолическую дисфункцию, у $42 \%$ сохраняются зоны локальной асинергии, в $16 \%$ формируется хроническая аневризма сердца. У каждого пятого молодого пациента наблюдается дезадаптивный тип ремоделирования сердца - эксцентрическая гипертрофия левого желудочка (ЛЖ) (табл. 2).

Таблица 2

Table 2

Эхокардиографические параметры у молодых пациентов через 12 месяцев после инфаркта миокарда

Echocardiographic parameters in young patients 12 months after myocardial infarction

\begin{tabular}{|c|c|}
\hline Параметр & Молодые пациенты с ИМ (n = 108) \\
\hline Конечно-диастолический размер, мм Ме (Q1-Q3) & $51,5(48,0-57,5)$ \\
\hline Конечно-систолический размер, мм Ме (Q1-Q3) & $35,0(33,0-42,0)$ \\
\hline Индекс конечно-диастолического размера, мм/м² $(\mathrm{M} \pm \mathrm{SD})$ & $27,0 \pm 3,5$ \\
\hline Конечно-диастолический объем, мл Ме (Q1-Q3) & $124,0(108,0-177,0)$ \\
\hline Конечно-систолический объем, мл Ме (Q1-Q3) & $52,0(43,0-79,0)$ \\
\hline $\begin{array}{l}\text { Индекс конечно-диастолического объема, мл/м² } \\
\text { Me (Q1-Q3) }\end{array}$ & $64,9(56,4-92,9)$ \\
\hline Индекс конечно-систолического объема, мл/м² Me (Q1-Q3) & $23,6(22,1-36,2)$ \\
\hline Диаметр левого предсердия, мм Me (Q1-Q3) & $37,0(34,0-40,0)$ \\
\hline Диаметр правого желудочка, мм Me (Q1-Q3) & $26,0(24,0-28,0)$ \\
\hline Межжелудочковая перегородка, мм Me (Q1-Q3) & $11,0(9,0-12,0)$ \\
\hline Задняя стенка ЛЖ, мм (M \pm SD) & $10,7 \pm 1,5$ \\
\hline Индекс массы миокарда ЛЖ, г/м² $(\mathrm{M} \pm \mathrm{SD})$ & $105,3 \pm 22,7$ \\
\hline Гипертрофия левого желудочка, \% & 14,8 \\
\hline Относительная толщина стенок Me (Q1-Q3) & $0,4(0,4-0,5)$ \\
\hline Зоны асинергии, \% & 41,9 \\
\hline Аневризма ЛЖ, \% & 16,3 \\
\hline Фракция выброса, \% Me (Q1-Q3) & $58,0(52,0-62,0)$ \\
\hline E/a Me (Q1-Q3) & $0,9(0,8-1,1)$ \\
\hline Систолическая дисфункция, \% & 18,0 \\
\hline Диастолическая дисфункция, \% & 59,6 \\
\hline Нормальная геометрия ЛЖ, \% & 40,0 \\
\hline Концентрическое ремоделирование ЛЖ, \% & 23,3 \\
\hline Концентрическая гипертрофия ЛЖ, \% & 16,7 \\
\hline Эксцентрическая гипертрофия ЛЖ, \% & 20,0 \\
\hline
\end{tabular}


У молодых пациентов на амбулаторном этапе реабилитации отмечается высокая среднесуточная ЧСС, часто встречаются желудочковые нарушения ритма: частая желудочковая экстрасистолия регистрируется у $30 \%$ пациентов, а эпизоды неустойчивой желудочковой тахикардии - у каждого десятого. В 12,5 \% случаев сохраняются эпизоды ишемической депрессии ST (табл. 3).

Таблица 3

Table 3

Параметры ХМ-ЭКГ у молодых пациентов через 12 месяцев после инфаркта миокарда Holter monitor parameters in young patients 12 months after myocardial infarction

\begin{tabular}{|l|c|}
\hline \multicolumn{1}{|c|}{ Параметр } & Молодые пациенты с ИМ (n = 108) \\
\hline Средняя ЧСС, уд/мин (M \pm SD) & $72,5 \pm 6,7$ \\
\hline Максимальная ЧСС, уд/мин (M \pm SD) & $119,1 \pm 13,8$ \\
\hline Минимальная ЧСС, уд/мин (M \pm SD) & $50,9 \pm 6,2$ \\
\hline Частая наджелудочковая экстрасистолия, \% & 5,9 \\
\hline Частая желудочковая экстарсистолия, \% & 30,3 \\
\hline Наджелудочковая тахикардия, \% & 4,5 \\
\hline Желудочковая тахикардия, \% & 9,1 \\
\hline Ишемическая депрессия сегмента ST, \% & 12,5 \\
\hline
\end{tabular}

На амбулаторном этапе реабилитации у большей части молодых пациентов имеются структурно-функциональные нарушения сердца. Нарушение механики ЛЖ признано важным фактором, способствующим прогрессирующему ишемическому ремоделированию, которое, в свою очередь, увеличивает риск развития желудочковых нарушений ритма [St. John Sutton et al., 2003; D’Elia et al., 2015]. Наличие рубцовой ткани может объяснять электрическую нестабильность миокарда. Патофизиологические изменения, происходящие в зоне инфаркта, включающие некроз кардиомиоцитов и замещение внеклеточного матрикса фиброзной тканью, приводят к возникновению гетерогенного субстрата с областями, обладающими потенциально медленной проводимостью, что индуцирует развитие рецидивирующей желудочковой аритмии [Leon et al., 2019]. По данным нашего исследования зоны локальной асинергии несмотря на проведенную реваскуляризацию сохраняются почти у половины больных, в $16 \%$ случаев формируется хроническая аневризма сердца. Хроническая аневризма является серьезным осложнением после ИМ, вызванные ей геометрические изменения еще больше ухудшают сократимость и способность к наполнению ЛЖ [Tavakoli et al., 2002; Lundblad et al., 2004]. Развитие сердечной недостаточности тесно связано с ремоделированием сердца [Argenziano et al., 2019]. Низкая фракция выброса в нашем исследовании встречалась у каждого пятого пациента. Доказано, что у лиц с низкой фракцией выброса желудочковые нарушения ритма встречаются чаще, чем при наличии сохраненной фракции выброса [Коваленко, Чичкова, 2013; Brembilla-Perrot et al., 2014; Кузнецов и др., 2015]. Установлено, что частота развития желудочковых аритмий обратно пропорциональна фракции выброса [Bhar-Amato, 2017]. Нарушение диастолической функции после ИМ по нашим данным имеют 60 \% молодых пациентов. Недавнее исследование показало, что диастолическая дисфункция также может являться предиктором развития желудочковых аритмий [Biering-Sorensen et al., 2016].

\section{Выводы}

Таким образом, в условиях амбулаторной реабилитации на фоне широкого распространения традиционных факторов риска (курения, дислипидемии, артериальной гипертензии, отягощенной по ранней ИБС наследственности, сахарного диабета 2 типа) у каждого пятого молодого пациента по данным эхокардиографии наблюдается неблагоприятное постинфарктное ремоделирование сердца - эксцентрическая гипертрофия ЛЖ. Нормальную 
геометрию ЛЖ имеют лишь 40 \% больных. В $23 \%$ случаев отмечается концентрическое ремоделирование ЛЖ, в 17 \% случаев - концентрическая гипертрофия ЛЖ. Зоны локальной гипо- и акинезии сохраняются почти у половины больных. У 15 \% молодых пациентов выявлена гипертрофия ЛЖ. В 60 \% случаев отмечается диастолическая, а в 18 \% случаев - систолическая дисфункция ЛЖ. У 16,3 \% пациентов сформировалась хроническая аневризма сердца. На амбулаторном этапе на фоне широкого распространения структурных нарушений сердца у трети молодых пациентов регистрируется частая желудочковая экстрасистолия, у каждого десятого - жизнеугрожающая желудочковая тахикардия. Через 12 месяцев не были достигнуты целевые значения среднесуточной ЧСС, у 12,5 \% пациентов по данным ХМ-ЭКГ сохранялись ишемические эпизоды. Результаты исследования представляют негативный прогноз у молодых больных после перенесенного ИМ.

\section{Конфликт интересов}

Автор заявляет об отсутствии конфликта интересов.

\section{Список литературы}

1. Коваленко Н.В., Чичкова М.А. 2013. Аритмогенная активность сердца при различных локализациях Q-инфаркта миокарда. Астраханский медицинский журнал, 8 (4): 76-79.

2. Кузнецов В.А., Тодосийчук В.В., Юркина Ю.А., Лыкасова Е.А., Нохрина О.Ю., Криночкин Д.В., Колунин Г.В., Горбатенко Е.А., Визнер Л.Р., Кутрунов В.Н., Дьячков С.М. 2015. Прогнозирование риска развития желудочковых аритмий высоких градаций у пациентов, направленных на коронарную ангиографию. Сибирский медицинский журнал, 30 (1): 105-111.

3. Пракопчик И.В., Гуревич О.В. 2008. Методы определения неблагоприятного прогноза после инфаркта миокарда. Вестник Смоленской государственной медицинской академии, 1: 50-52.

4. Argenziano M.A., Doss M.X., Tabler M., Sachinidis A., Antzelevitch C. 2019. Transcriptional changes associated with advancing stages of heart failure underlie atrial and ventricular arrhythmogenesis. PLoS One, 14 (5): e0216928.

5. Bhar-Amato J., Davies W., Agarwal S. 2017. Ventricular Arrhythmia after Acute Myocardial Infarction: «The Perfect Storm». Arrhythm. Electrophysiol. Rev., 6 (3): 134-139.

6. Biering-Sorensen T., Olsen F.J., Storm K., Fritz-Hansen T., Olsen N.T., Jons C., Vinther M., Sogaard P., Risum N. 2016. Prognostic value of tissue Doppler imaging for predicting ventricular arrhythmias and cardiovascular mortality in ischaemic cardiomyopathy. Eur. Heart. J. Cardiovasc. Imaging, 17 (7): 722-731.

7. Brembilla-Perrot B., Huttin O., Azman B., Sellal J.M., Schwartz J., Olivier A., Blangy H., Sadoul N. 2014. Influence of the mode of management of acute myocardial infarction on the inducibility of ventricular tachyarrhythmias with programmed ventricular stimulation after myocardial infarction. Isr. Med. Assoc. J., 16 (6): 352-357.

8. Chang P.C., Lin S.F., Chu Y., Wo H.T., Lee H.L., Huang Y.C., Wen M.S., Chou C.C. 2019. LCZ696 Therapy Reduces Ventricular Tachyarrhythmia Inducibility in a Myocardial Infarction-Induced Heart Failure Rat Model. Cardiovasc. Ther., 2019.

9. D’Elia N., D’hooge J., Marwick T.H. 2015. Association Between Myocardial Mechanics and Ischemic LV Remodeling. JACC Cardiovasc. Imaging, 8 (12): 1430-1443.

10. Jons C., Sogaard P., Behrens S., Schrader J., Mrosk S., Bloch Thomsen P.E. 2019. The clinical effect of arrhythmia monitoring after myocardial infarction (BIO-GUARD MI): study protocol for a randomized controlled trial. Trials, 20 (1): 563.

11. Leon D.G., Lopez-Yunta M., Alfonso-Almazán J.M., Marina-Breysse M., Quintanilla J.G., Sanchez-Gonzalez J., Galan-Arriola C., Castro-Nunez F., Gonzalez-Ferrer J.J., Ibanez B., PerezVillacastin J., Perez-Castellano N., Fuster V., Jalife J., Vazquez M., Aguado-Sierra J., FilgueirasRama D. 2019. Three-dimensional cardiac fibre disorganization as a novel parameter for ventricular arrhythmia stratification after myocardial infarction. Europace, 21 (5): 822-832.

12. Lundblad R., Abdelnoor M., Svennevig J.L. 2004. Surgery for left ventricular aneurysm: early and late survival after simple linear repair and endoventricular patch plast. J. Thorac. Cardiovasc. Surg., 128: 449-456. 
13. McMurray J., Kober L., Robertson M., Dargie H., Colucci W., Lopez-Sendon J., Remme W., Sharpe D.N., Ford I. 2005. Antiarrhythmic effect of carvedilol after acute myocardial infarction: Results of the carvedilol post-infarct survival control in left ventricular dysfunction (CAPRICORN) trial. Journal of the American College of Cardiology, 45 (4): 25-530.

14. Risgaard B., Nielsen J.B., Jabbari R., Haunso S., Holst A.G., Winkel B.G. 2013. Prior myocardial infarction in the young: predisposes to a high relative risk but low absolute risk of a sudden cardiac death. Europace, 15: 48-54.

15. Setoguchi S., Glynn R.J., Avorn J. 2008. Improvements in long-term mortality after myocardial infarction and increased use of cardiovascular drugs after discharge: a 10-year trend analysis. J. Am. Coll. Cardiol., 51: 1247-1254.

16. Solomon S.D., Zelenkofske S., McMurray J.J., Finn P.V., Velazquez E., Ertl G., Harsanyi A., Rouleau J.L., Maggioni A., Kober L., White H., Van de Werf F., Pieper K., Califf R.M., Pfeffer M.A. 2005. Sudden death in patients with myocardial infarction and left ventricular dysfunction, heart failure, or both. N. Engl. J. Med., 352: 2581-2588.

17. St. John Sutton M., Lee D., Rouleau J.L., Goldman S., Plappert T., Braunwald E., Pfeffer M.A. 2003. Left ventricular remodeling and ventricular arrhythmias after myocardial infarction. Circulation, 107 (20): 2577-2582.

18. Tavakoli R., Bettex D., Weber A., Brunner H., Genoni M., Pretre R., Jenni R., Turina M. 2002. Repair of postinfarction dyskinetic LV aneurysm with either linear or patch technique. European journal of cardio-thoracic surgery: official journal of the European Association for Cardio-thoracic Surgery, 22 (1): 129-134.

19. Tomaselli G.F., Zipes D.P. 2004. What causes sudden death in heart failure? Circ Res, 95: 754-763.

20. World Health Organization. The top 10 causes of death. 2014. Available: http://www.who.int/mediacentre/factsheets/fs310/en/.

\section{References}

1. Kovalenko N.V., Chichkova M.A. 2013. Aritmogennaya aktivnost' serdca pri razlichnyh lokalizaciyah Q-infarkta miokarda [The arrhythmogenic activity of the heart in different location of Qmyocardial infarction]. Astrahanskij medicinskij zhurnal, 8 (4): 76-79.

2. Kuznecov V.A., Todosijchuk V.V., Yurkina Yu.A., Lykasova E.A., Nohrina O.Yu., Krinochkin D.V., Kolunin G.V., Gorbatenko E.A., Vizner L.R., Kutrunov V.N., D'yachkov S.M. 2015. Prognozirovanie riska razvitiya zheludochkovyh aritmij vysokih gradacij u pacientov, napravlennyh na koronarnuyu angiografiyu [Prediction of high-grade ventricular arrhythmias in patients referred for the coronary angiography]. Sibirskij medicinskij zhurnal, 30 (1): 105-111.

3. Prakopchik I.V., Gurevich O.V. 2008. Metody opredeleniya neblagopriyatnogo prognoza posle infarkta miokarda [Methods for determining the unfavorable prognosis after myocardial infarction]. Vestnik Smolenskoj gosudarstvennoj medicinskoj akademii, 1: 50-52.

4. Argenziano M.A., Doss M.X., Tabler M., Sachinidis A., Antzelevitch C. 2019. Transcriptional changes associated with advancing stages of heart failure underlie atrial and ventricular arrhythmogenesis. PLoS One, 14 (5): e0216928.

5. Bhar-Amato J., Davies W., Agarwal S. 2017. Ventricular Arrhythmia after Acute Myocardial Infarction: «The Perfect Storm». Arrhythm. Electrophysiol. Rev., 6 (3): 134-139.

6. Biering-Sorensen T., Olsen F.J., Storm K., Fritz-Hansen T., Olsen N.T., Jons C., Vinther M., Sogaard P., Risum N. 2016. Prognostic value of tissue Doppler imaging for predicting ventricular arrhythmias and cardiovascular mortality in ischaemic cardiomyopathy. Eur. Heart. J. Cardiovasc. Imaging, 17 (7): 722-731.

7. Brembilla-Perrot B., Huttin O., Azman B., Sellal J.M., Schwartz J., Olivier A., Blangy H., Sadoul N. 2014. Influence of the mode of management of acute myocardial infarction on the inducibility of ventricular tachyarrhythmias with programmed ventricular stimulation after myocardial infarction. Isr. Med. Assoc. J., 16 (6): 352-357.

8. Chang P.C., Lin S.F., Chu Y., Wo H.T., Lee H.L., Huang Y.C., Wen M.S., Chou C.C. 2019. LCZ696 Therapy Reduces Ventricular Tachyarrhythmia Inducibility in a Myocardial Infarction-Induced Heart Failure Rat Model. Cardiovasc. Ther., 2019. 
9. D'Elia N., D'hooge J., Marwick T.H. 2015. Association Between Myocardial Mechanics and Ischemic LV Remodeling. JACC Cardiovasc. Imaging, 8 (12): 1430-1443.

10. Jons C., Sogaard P., Behrens S., Schrader J., Mrosk S., Bloch Thomsen P.E. 2019. The clinical effect of arrhythmia monitoring after myocardial infarction (BIO-GUARD MI): study protocol for a randomized controlled trial. Trials, 20 (1): 563.

11. Leon D.G., Lopez-Yunta M., Alfonso-Almazán J.M., Marina-Breysse M., Quintanilla J.G., Sanchez-Gonzalez J., Galan-Arriola C., Castro-Nunez F., Gonzalez-Ferrer J.J., Ibanez B., PerezVillacastin J., Perez-Castellano N., Fuster V., Jalife J., Vazquez M., Aguado-Sierra J., FilgueirasRama D. 2019. Three-dimensional cardiac fibre disorganization as a novel parameter for ventricular arrhythmia stratification after myocardial infarction. Europace, 21 (5): 822-832.

12. Lundblad R., Abdelnoor M., Svennevig J.L. 2004. Surgery for left ventricular aneurysm: early and late survival after simple linear repair and endoventricular patch plast. J. Thorac. Cardiovasc. Surg., 128: 449-456.

13. McMurray J., Kober L., Robertson M., Dargie H., Colucci W., Lopez-Sendon J., Remme W., Sharpe D.N., Ford I. 2005. Antiarrhythmic effect of carvedilol after acute myocardial infarction: Results of the carvedilol post-infarct survival control in left ventricular dysfunction (CAPRICORN) trial. Journal of the American College of Cardiology, 45 (4): 25-530.

14. Risgaard B., Nielsen J.B., Jabbari R., Haunso S., Holst A.G., Winkel B.G. 2013. Prior myocardial infarction in the young: predisposes to a high relative risk but low absolute risk of a sudden cardiac death. Europace, 15: 48-54.

15. Setoguchi S., Glynn R.J., Avorn J. 2008. Improvements in long-term mortality after myocardial infarction and increased use of cardiovascular drugs after discharge: a 10-year trend analysis. J. Am. Coll. Cardiol., 51: 1247-1254.

16. Solomon S.D., Zelenkofske S., McMurray J.J., Finn P.V., Velazquez E., Ertl G., Harsanyi A., Rouleau J.L., Maggioni A., Kober L., White H., Van de Werf F., Pieper K., Califf R.M., Pfeffer M.A. 2005. Sudden death in patients with myocardial infarction and left ventricular dysfunction, heart failure, or both. N. Engl. J. Med., 352: 2581-2588.

17. St. John Sutton M., Lee D., Rouleau J.L., Goldman S., Plappert T., Braunwald E., Pfeffer M.A. 2003. Left ventricular remodeling and ventricular arrhythmias after myocardial infarction. Circulation, 107 (20): 2577-2582.

18. Tavakoli R., Bettex D., Weber A., Brunner H., Genoni M., Pretre R., Jenni R., Turina M. 2002. Repair of postinfarction dyskinetic LV aneurysm with either linear or patch technique. European journal of cardio-thoracic surgery: official journal of the European Association for Cardio-thoracic Surgery, 22 (1): 129-134.

19. Tomaselli G.F., Zipes D.P. 2004. What causes sudden death in heart failure? Circ Res, 95: 754-763.

20. World Health Organization. The top 10 causes of death. 2014. Available: http://www.who.int/mediacentre/factsheets/fs310/en/.

\section{ИНФОРМАЦИЯ ОБ АВТОРАХ}

Новикова кардиолог отделения Федерального центра сердечнососудистой хирургии имени С.Г. Суханова, г. Пермь, Россия

Некрутенко Людмила Александровна, доктор медицинских наук, профессор кафедры госпитальной терапии и кардиологии Пермского государственного медицинского университета имени академика Е.А. Вагнера, г. Пермь, Россия

\section{INFORMATION ABOUT THE AUTHORS}

Irina A. Novikova, cardiologist of the admissionpolyclinic department of the Federal Center for Cardiovascular Surgery named after S.G. Sukhanov, Perm, Russia

Ludmila A. Nekrutenko, Doctor of Medical Sciences, Professor of the Department of Hospital Therapy and Cardiology, Perm State Medical University named after Academician E.A. Wagner, Perm, Russia 
Василец Любовь Михайловна, доктор медицинских наук, профессор кафедры госпитальной терапии и кардиологии Пермского государственного медицинского университета имени академика Е.А. Вагнера, г. Пермь, Россия

Шишкина Екатерина Андреевна, кандидат медицинских наук, доцент кафедры госпитальной терапии и кардиологии Пермского государственного медицинского университета имени академика Е.А. Вагнера, г. Пермь, Россия

Родионов Роман Андреевич,

ординатор кафедры госпитальной терапии и кардиологии Пермского государственного медицинского университета имени академика Е.А. Вагнера, г. Пермь, Россия
Liubov M. Vasilets, Doctor of Medical Sciences, Professor of the Department of Hospital Therapy and Cardiology, Perm State Medical University named after Academician E.A. Wagner, Perm, Russia

Ekaterina A. Shishkina, Candidate of Medical Sciences, Associate Professor of the Department of Hospital Therapy and Cardiology, Perm State Medical University named after Academician E.A. Wagner, Perm, Russia

Roman A. Rodionov, Resident of the Department of Hospital Therapy and Cardiology, Perm State Medical University named after academician E.A. Wagner, Perm, Russia 